\title{
Iterating Fixed Point via Generalized Mann's Iteration in Convex b-Metric Spaces with Application
}

\author{
A. Asif $\mathbb{D}^{1},{ }^{1}$ M. Alansari, ${ }^{2}$ N. Hussain $\left(\mathbb{D},{ }^{2}\right.$ M. Arshad $\left(\mathbb{D},{ }^{1}\right.$ and A. Ali ${ }^{1}$ \\ ${ }^{1}$ Department of Mathematics and Statistics, International Islamic University, Islamabad 44000, Pakistan \\ ${ }^{2}$ Department of Mathematics, King Abdulaziz University, P.O. Box 80203, Jeddah 21589, Saudi Arabia \\ Correspondence should be addressed to A. Asif; awais.asif21@gmail.com and N. Hussain; nhusain@kau.edu.sa
}

Received 30 June 2020; Revised 6 February 2021; Accepted 12 February 2021; Published 27 February 2021

Academic Editor: Danilo Comminiello

Copyright ( 2021 A. Asif et al. This is an open access article distributed under the Creative Commons Attribution License, which permits unrestricted use, distribution, and reproduction in any medium, provided the original work is properly cited.

This manuscript investigates fixed point of single-valued Hardy-Roger's type F-contraction globally as well as locally in a convex $b$-metric space. The paper, using generalized Mann's iteration, iterates fixed point of the abovementioned contraction; however, the third axiom (F3) of the $F$-contraction is removed, and thus the mapping $F$ is relaxed. An important approach used in the article is, though a subset closed ball of a complete convex $b$-metric space is not necessarily complete, the convergence of the Cauchy sequence is confirmed in the subset closed ball. The results further lead us to some important corollaries, and examples are produced in support of our main theorems. The paper most importantly presents application of our results in finding solution to the integral equations.

\section{Introduction and Preliminaries}

Various authors generalized metric space into many interesting generalizations (see [1-8]). Among them, Czerwik [9] introduced the idea of $b$-metric and proved fixed point theorems in it. It was further extended to partial b-metric and dislocated b-metric spaces in the past years $[10,11]$. Chen et al. [12] introduced convex $b$-metric space and established various fixed point theorems.

On the contrary, different authors extended Banach contraction to its generalizations (see [2-17]). Wardowski [18] introduced the idea of $F$-contraction which was later followed by many authors who delivered interesting results of $F$-contraction. One of them was presented by Cosentino et al. [19] who expanded $F$-contraction in $F$-contraction of Hardy-Roger's type.

In this article, we discuss $F$-contraction in the frame of convex b-metric space using generalized Mann's iteration algorithm. However, we have modified definition of $F$-contraction by eliminating two of its conditions and define $F$-Hardy-Roger's contraction on closed ball in convex b-metric space. A detailed example explains the proved results.
Some fundamental definitions related to our work are given below.

Definition 1 (see [20]). Let $k \geq 1$ be a real number. We denote by $\mathscr{F}$ the family of all functions.

$F: R^{+} \longrightarrow R$ with the following properties:

(F1) $F$ is strictly increasing.

(F2) for each sequence $\left\{a_{n}\right\} \subset R^{+}, \lim _{n \rightarrow \infty} a_{n}=0$ if and only if $\lim _{n \rightarrow \infty} F\left(a_{n}\right)=-\infty$.

(F3) for each sequence $\left\{a_{n}\right\} \subset R^{+}, \lim _{n \rightarrow \infty} a_{n}=0$, there exists $m \in(0,1)$ such that $\lim _{n \rightarrow \infty}\left(a_{n}\right)^{m}$ $F\left(a_{n}\right)=0$.

(F4) for each sequence $\left(a_{n}\right) \subset R^{+}$of positive numbers such that $\tau+F\left(k a_{n}\right) \leq F\left(k a_{n-1}\right)$ for all $n \in N$ and some $\tau \in R^{+}$, then $\tau+F\left(k^{n} a_{n}\right) \leq F\left(k^{n-1} a_{n-1}\right)$ for all $\mathrm{n} \in N$.

Definition 2 (see [20]). Let $(E, d, k)$ be a b-metric space where $k \geq 1$. A multivalued mapping $T: E \longrightarrow C B(E)$ is called an $F$-contraction of Nadler type if there exist $F \in \mathscr{F}_{s}$ 
and $\tau \in R^{+}$such that $\tau+F(k H(T a, T b)) \leq F d(a, b)$ for all $a, b \in E$ with $T a=T b$.

Note that, in our theorems, we will consider $\mathscr{F}_{b}$ as the class of functions satisfying only (F1) and (F2) which modifies the definition of $F$-contraction.

Definition 3 (see [9]). Assume that $E=\varnothing$ with $1 \leq k \in R$. If $b_{k}: E \times E \longrightarrow[0,+\infty)$ satisfies the following axioms, for each $a, b, c \in E$.

(1) $b_{k}(a, b)=0$ if and only if $a=b$.

(2) $b_{k}(a, b)=b_{k}(b, a)$.

(3) $b_{k}(a, b) \leq k\left[b_{k}(a, c)+b_{k}(c, b)\right]$. $k \geq 1$.

Then the pair $\left(E, b_{k}\right)$ is known as b-metric space with

Definition 4 (see [9]). Suppose $\left(a_{n}\right)$ is a sequence in $E$. Then

(1) $\left(a_{n}\right)$ is convergent to a point $a \in E$ if $\lim _{n \longrightarrow \infty} d\left(a_{n}, a\right)=0$.

(2) $\left(a_{n}\right)$ is Cauchy if $\lim _{n, m \longrightarrow \infty} d\left(a_{n}, a_{m}\right)=0$.

(3) The space $\left(E, b_{k}\right)$ is complete if every Cauchy sequence $\left(a_{n}\right) \subset E$ is convergent to a point $a \in E$.

Definition 5 (see [12]). Assume that $E=\varnothing$ and $b_{k}: E \times$ $E \longrightarrow[0,+\infty)$ is a mapping. Let $I=[0,1]$ with a continuous mapping $\eta: E \times E \times I \longrightarrow E$ such that

$$
b_{k}(o, \eta(a, b ; \delta)) \leq \delta b_{k}(o, a)+(1-\delta) b_{k}(o, b),
$$

for each $o \in E$ and $(a, b, \delta) \in E \times E \times I . \eta$ is said to be a convex structure on $E$.

Definition 6 (see [12]). Assume that $\eta: E \times E \times I \longrightarrow E$ is a convex structure on a b-metric space $\left(E, b_{k}\right)$. Then $\left(E, b_{k}, \eta\right)$ is known as convex $b$-metric space.

Suppose that $\left(E, b_{k}, \eta\right)$ is convex b-metric space with a self-mapping $f$. Then for $a_{n} \in E$ and $\delta_{n} \in[0,1]$, a generalize Mann's iteration sequence $\left\{a_{n}\right\}$ is defined as

$$
a_{n+1}=\eta\left(a_{n}, f a_{n} ; \delta_{n}\right), \quad n \in N .
$$

Definition 7 (see [12]). Let $(E, d)$ be a metric space. A selfmapping $f$ on $E$ is called an $F$-contraction of Hardy-Roger's type if there exist $F \in \mathscr{F}^{\prime}$ and $\tau \in R^{+}$such that

$$
\begin{aligned}
\tau+F(d(f a, f b)) \leq & F(\alpha d(a, b y)+\beta d(a, f a) \\
& +\gamma d(b, f b)+\delta d(a, f b) \\
& +L d(b, T a)),
\end{aligned}
$$

for all $a, b \in E$ with $d(f a, f b)>0$, where $\alpha+\beta+\gamma+2 \delta=1$, $\gamma \neq 1$, and $L \geq 0$.

In the above definition, $\mathscr{F}^{\prime}$ is the class of functions that satisfies (F1)-(F3).

\section{Fixed Point Results of $F$-Hardy-Roger's Contractions on Closed Ball}

This section examines F-Hardy-Roger's contraction for fixed point while executing the contraction only on closed ball rather than the whole convex b-metric. Also, an example is given to explain the proved theorem.

Note that, in our theorems, we will consider $\mathscr{F}_{b}$ as the class of functions satisfying only (F1) and (F2) which modifies the definition of $F$-contraction and $I=[0,1]$.

Definition 8. Let $\left(E, b_{k}, \eta\right)$ is a convex b-metric space, $a_{0}$ is some element in $E$, and $\varepsilon>0$; then the set $S_{\varepsilon}\left[a_{0}\right]=\left\{a \in E: b_{k}\left(a_{0}, a\right) \leq \varepsilon\right\}$ is called a closed ball in $E$.

Not that, $S_{\varepsilon}\left[a_{0}\right]$ is a nonempty subset containing at least one element $a_{0}$. Also, a subset of a complete b-metric space may not be complete. Hence a Cauchy sequence in the subset closed ball of a complete b-metric space may not converge in closed ball.

Definition 9. Assume that $F \in \mathscr{F}_{b},\left(E, b_{k}, \eta\right)$ be a convex $b$ metric space with $k>1, \delta_{n-1} \in[0,1)$ for $n \in N$, and $S_{\varepsilon}\left[a_{0}\right]$ be a closed ball in $E$. Then $f: E \longrightarrow E$ is known as F-HardyRoger's contraction on closed ball if for $\alpha, \beta, \gamma \in[0, \infty)$ with $k\left(k^{2} \delta_{n-1}+\alpha k\right)+\left(k^{2}+1\right)(\beta+2 \gamma k)<1$, the following hold:

$$
\begin{aligned}
\tau+F\left(k b_{k}(f a, f b)\right) \leq & F\left[\alpha b_{k}(a, b)+\beta\left\{b_{k}(a, f a)+b_{k}(b, f b)\right\}\right. \\
& \left.+\gamma\left\{b_{k}(a, f b)+b_{k}(b, f a)\right\}\right],
\end{aligned}
$$

for every $a, b \in E$.

Theorem 1. Suppose $\left(E, b_{k}, \eta\right)$ is a complete convex b-metric space with generalized Mann's iteration $\left\{a_{n}\right\}$ having $\delta_{n-1} \in\left(0,\left(1 / 4 k^{2}\right]\right)$. Suppose $f: E \longrightarrow E$ is an F-HardyRoger's contraction on closed ball, and for all $n \in N, \lambda=$ $\left(\left(k \delta_{n-1}+\alpha+\beta+k \gamma+\gamma \delta_{n-1}\right) /(1-\beta-k \gamma)\right)$ and the following holds:

$$
b_{k}\left(a_{0}, f a_{0}\right) \leq \lambda(1-k \lambda) \varepsilon .
$$

Then $f$ has a unique fixed point in $E$.

Proof. First we prove that the iteration $\left\{a_{n}\right\}$ belongs to the closed ball. To do so, we will use mathematical induction. Since

$$
\begin{aligned}
b_{k}\left(a_{0}, a_{1}\right)= & b_{k}\left(a_{0}, \eta\left(a_{0}, f a_{0} ; \delta_{0}\right)\right) \leq\left(1-\delta_{0}\right) b_{k}\left(a_{0}, f a_{0}\right) \\
& \leq\left(1-\delta_{0}\right) \lambda(1-k \lambda) \varepsilon<\varepsilon
\end{aligned}
$$

that is, $b_{k}\left(a_{0}, a_{1}\right)<\varepsilon$, therefore $a_{1} \in S_{\varepsilon}\left[a_{0}\right]$. Suppose $a_{2}, a_{3}, \ldots, a_{m} \in S_{\varepsilon}\left[a_{0}\right]$. Now for $a_{m+1}$, as

$$
\begin{aligned}
& b_{k}\left(a_{m}, a_{m+1}\right) \leq\left(1-\delta_{m}\right) b_{k}\left(a_{m}, f a_{m}\right), \\
& b_{k}\left(a_{m}, f a_{m}\right) \leq k b_{k}\left(a_{m}, f a_{m-1}\right)+k b_{k}\left(f a_{m-1}, f a_{m}\right) .
\end{aligned}
$$

Then by (4), 


$$
\tau+F\left(k b_{k}\left(f a_{m-1}, f a_{m}\right)\right) \leq F\left[\alpha b_{k}\left(a_{m-1}, a_{m}\right)+\beta\left\{b_{k}\left(a_{m-1}, f a_{m-1}\right)+b_{k}\left(a_{m}, f a_{m}\right)\right\}+\gamma\left\{b_{k}\left(a_{m-1}, f a_{m}\right)+b_{k}\left(a_{m}, f a_{m-1}\right)\right\}\right],
$$

and this implies that

$$
\begin{aligned}
F\left(b_{k}\left(a_{m}, f a_{m}\right)\right)= & F\left(k b_{k}\left(a_{m}, f a_{m-1}\right)+k b_{k}\left(f a_{m-1}, f a_{m}\right)\right) \\
\leq & F\left[k b_{k}\left(a_{m}, f a_{m-1}\right)+\alpha\left(1-\delta_{m-1}\right) b_{k}\left(a_{m-1}, f a_{m-1}\right)+\beta\left\{b_{k}\left(a_{m-1}, f a_{m-1}\right)+b_{k}\left(a_{m}, f a_{m}\right)\right\}\right. \\
& \left.+\gamma\left\{b_{k}\left(a_{m-1}, f a_{m}\right)+b_{k}\left(a_{m}, f a_{m-1}\right)\right\}\right]-\tau \\
\leq & F\left[k \delta_{m-1} b_{k}\left(a_{m-1}, f a_{m-1}\right)+\alpha\left(1-\delta_{m-1}\right) b_{k}\left(a_{m-1}, f a_{m-1}\right)+\beta\left\{b_{k}\left(a_{m-1}, f a_{m-1}\right)+b_{k}\left(a_{m}, f a_{m}\right)\right\}\right. \\
& \left.+\gamma\left\{k b_{k}\left(a_{m-1}, a_{m}\right)+k b_{k}\left(a_{m}, f a_{m}\right)+\delta_{m-1} b_{k}\left(a_{m-1}, f a_{m-1}\right)\right\}\right]-\tau \\
\leq & F\left[k \delta_{m-1} b_{k}\left(a_{m-1}, f a_{m-1}\right)+\alpha\left(1-\delta_{m-1}\right) b_{k}\left(a_{m-1}, f a_{m-1}\right)+\beta\left\{b_{k}\left(a_{m-1}, f a_{m-1}\right)+b_{k}\left(a_{m}, f a_{m}\right)\right\}\right. \\
& \left.+\gamma\left\{k\left(1-\delta_{m-1}\right) b_{k}\left(a_{m-1}, f a_{m-1}\right)+k b_{k}\left(a_{m}, f a_{m}\right)+\delta_{m-1} b_{k}\left(a_{m-1}, f a_{m-1}\right)\right\}\right]-\tau .
\end{aligned}
$$

Using (F1), we write

$$
\begin{aligned}
(1-\beta-k \gamma) b_{k}\left(a_{m}, f a_{m}\right) \leq & \left(k \delta_{m-1}+\alpha\left(1-\delta_{m-1}\right)+\beta\right. \\
& \left.+k\left(1-\delta_{m-1}\right) \gamma+\gamma \delta_{m-1}\right) \\
& \cdot b_{k}\left(a_{m-1}, f a_{m-1}\right),
\end{aligned}
$$

and this implies that

$$
\begin{aligned}
b_{k}\left(a_{m}, f a_{m}\right) & <\frac{\left(k \delta_{m-1}+\alpha+\beta+k \gamma+\gamma \delta_{m-1}\right)}{(1-\beta-k \gamma)} b_{k}\left(a_{m-1}, f a_{m-1}\right) \\
& =\lambda b_{k}\left(a_{m-1}, f a_{m-1}\right),
\end{aligned}
$$

that is, for all $m \in N$,

$$
b_{k}\left(a_{m}, f a_{m}\right)<\lambda b_{k}\left(a_{m-1}, f a_{m-1}\right) .
$$

Note that $\lambda<1$. Consequently,

$$
\begin{aligned}
b_{k}\left(a_{m}, f a_{m}\right) & <\lambda b_{k}\left(a_{m-1}, f a_{m-1}\right)<\lambda b_{k}\left(a_{m-2}, f a_{m-2}\right) \\
& <\cdots<\lambda b_{k}\left(a_{0}, f a_{0}\right),
\end{aligned}
$$

and hence $b_{k}\left(a_{m}, f a_{m}\right)<\lambda^{m} b_{k}\left(a_{0}, f a_{0}\right)$ for all $m \in N$. Thus by (7), $b_{k}\left(a_{m}, a_{m+1}\right)<\lambda^{m} b_{k}\left(a_{0}, f a_{0}\right)$ for all $m \in N$. By triangular inequality

$$
\begin{aligned}
b_{k}\left(a_{0}, a_{m+1}\right) & \leq k b_{k}\left(a_{0}, a_{1}\right)+k^{2} b_{k}\left(a_{1}, a_{2}\right)+\cdots+k^{m+1} b_{k}\left(a_{m}, a_{m+1}\right) \\
& <k\left[b_{k}\left(a_{0}, a_{1}\right)+k \lambda b_{k}\left(a_{0}, a_{1}\right)+\cdots+(k \lambda)^{m} b_{k}\left(a_{0}, a_{1}\right)\right] \\
& =\frac{k\left(1-(k \lambda)^{m}\right)}{1-k \lambda} b_{k}\left(a_{0}, a_{1}\right) \\
& =\frac{k\left(1-(k \lambda)^{m}\right)}{1-k \lambda} \lambda(1-k \lambda) \varepsilon<\varepsilon .
\end{aligned}
$$

Hence $a_{m+1} \in S_{\varepsilon}\left[a_{0}\right]$. Therefore, we conclude that $a_{n} \in S_{\varepsilon}\left[a_{0}\right]$ for all $n \in N$. Now from (4), (7), and (12), we write

$$
\begin{aligned}
F\left(b_{k}\left(a_{n}, a_{n+1}\right)\right) & \leq F\left(b_{k}\left(a_{n-1}, f a_{n-1}\right)\right)-\tau \\
& \leq F\left(b_{k}\left(a_{n-2}, f a_{n-2}\right)\right)-2 \tau \leq \cdots \\
& \leq F\left(b_{k}\left(a_{0}, f a_{0}\right)\right)-n \tau .
\end{aligned}
$$

Exerting limit $n \longrightarrow \infty$, we obtain

$$
\lim _{n \longrightarrow \infty} F\left(b_{k}\left(a_{n}, f a_{n}\right)\right)=-\infty .
$$

Consequently by (F2), we acquire

$$
\lim _{n \longrightarrow \infty} b_{k}\left(a_{n}, f a_{n}\right)=0 .
$$

Hence, $\lim _{n \longrightarrow \infty} b_{k}\left(a_{n}, a_{n+1}\right)=\lim _{n \longrightarrow \infty} b_{k}\left(a_{n}, f a_{n}\right)=0$. It remains to prove that the sequence $\left\{a_{n}\right\}$ is a Cauchy sequence. Suppose on the contrary that $\left\{a_{n}\right\}$ is not Cauchy. Then we can find a positive real number $\varepsilon_{0}$ and two subsequences $\left\{a_{\mu(u)}\right\}$ and $\left\{a_{\omega(u)}\right\}$ of $\left\{a_{n}\right\}$ while $\mu(u)>\omega(u)>u$ with $\mu(u)$ as the smallest natural index such that 


$$
\begin{gathered}
b_{k}\left(a_{\mu(u)}, a_{\omega(u)}\right) \geq \sigma_{0}, \\
b_{k}\left(a_{\mu(u)-1}, a_{\omega(u)}\right)<\sigma_{0} .
\end{gathered}
$$

Using the above inequalities, we can write

$$
\begin{aligned}
\sigma_{0} & \leq b_{k}\left(a_{\mu(u)}, a_{\omega(u)}\right) \leq k\left[b_{k}\left(a_{\mu(u)}, a_{\omega(u)+1}\right)+b_{k}\left(a_{\omega(u)+1}, a_{\omega(u)}\right)\right] \frac{\sigma_{0}}{k} \\
& \leq \lim _{u \longrightarrow \infty} \sup _{k}\left(a_{\mu(u)}, a_{\omega(u)+1}\right) .
\end{aligned}
$$

Now,

$$
\begin{aligned}
b_{k}\left(a_{\mu(u)}, a_{\omega(u)+1}\right) & \leq b_{k}\left(\left(\eta a_{\mu(u)-1}, f a_{\mu(u)-1}, \delta_{\mu(u)-1}\right), a_{\omega(u)+1}\right) \\
& =\delta_{\mu(u)-1} b_{k}\left(a_{\mu(u)-1}, a_{\omega(u)+1}\right)+\left(1-\delta_{\mu(u)-1}\right) b_{k}\left(f a_{\mu(u)-1}, a_{\omega(u)+1}\right) \\
& \leq \delta_{\mu(u)-1} b_{k}\left(a_{\mu(u)-1}, a_{\omega(u)+1}\right)+\left(1-\delta_{\mu(u)-1}\right) k\left\{b_{k}\left(f a_{\mu(u)-1}, f a_{\omega(u)+1}\right)+b_{k}\left(f a_{\omega(u)+1}, a_{\omega(u)+1}\right)\right\} .
\end{aligned}
$$

Since, by (4),

$$
\begin{aligned}
& F\left(k b_{k}\left(f a_{\mu(u)-1}, f a_{\omega(u)+1}\right)\right) \\
& \leq F\left\{\alpha b_{k}\left(a_{\mu(u)-1}, a_{\omega(u)+1}\right)+\beta\left(b_{k}\left(a_{\mu(u)-1}, f a_{\mu(u)-1}\right)+b_{k}\left(f a_{\omega(u)+1}, a_{\omega(u)+1}\right)\right)\right. \\
& \left.\quad+\gamma\left(b_{k}\left(a_{\mu(u)-1}, f a_{\omega(u)+1}\right)+b_{k}\left(a_{\omega(u)+1}, f a_{\mu(u)-1}\right)\right)\right\}-\tau,
\end{aligned}
$$

and therefore,

$$
\begin{aligned}
& F\left[\delta_{\mu(u)-1} b_{k}\left(a_{\mu(u)-1}, a_{\omega(u)+1}\right)+\left(1-\delta_{\mu(u)-1}\right)\left\{k b_{k}\left(f a_{\mu(u)-1}, f a_{\omega(u)+1}\right)+k b_{k}\left(f a_{\omega(u)+1}, a_{\omega(u)+1}\right)\right\}\right] \\
& \leq F \\
& \quad\left[\delta_{\mu(u)-1} b_{k}\left(a_{\mu(u)-1}, a_{\omega(u)+1}\right)+\left(1-\delta_{\mu(u)-1}\right)\left\{\alpha b_{k}\left(a_{\mu(u)-1}, a_{\omega(u)+1}\right)\right.\right. \\
& \quad+\beta\left(b_{k}\left(a_{\mu(u)-1}, f a_{\mu(u)-1}\right)+b_{k}\left(f a_{\omega(u)+1}, a_{\omega(u)+1}\right)\right) \\
& \left.\left.\quad+\gamma\left(b_{k}\left(a_{\mu(u)-1}, f a_{\omega(u)+1}\right)+b_{k}\left(a_{\omega(u)+1}, f a_{\mu(u)-1}\right)\right)+k b_{k}\left(f a_{\omega(u)+1}, a_{\omega(u)+1}\right)\right\}\right]-\tau,
\end{aligned}
$$

using (F1) and (20), we write

$$
\begin{aligned}
& b_{k}\left(a_{\mu(u)}, a_{\omega(u)+1}\right) \\
& \leq \gamma_{\mu(u)-1} b_{k}\left(a_{\mu(u)-1}, a_{\omega(u)+1}\right) \\
&+\left(1-\delta_{\mu(u)-1}\right)\left\{\alpha b_{k}\left(a_{\mu(u)-1}, a_{\omega(u)+1}\right)+\beta\left(b_{k}\left(a_{\mu(u)-1}, f a_{\mu(u)-1}\right)+b_{k}\left(f a_{\omega(u)+1}, a_{\omega(u)+1}\right)\right)\right. \\
&+\gamma\left(b_{k}\left(a_{\mu(u)-1}, f a_{\omega(u)+1}\right)+b_{k}\left(a_{\omega(u)+1}, f a_{\mu(u)-1}\right)\right)+k b_{k}\left(f a_{\omega(u)+1}, a_{\omega(u)+1}\right) \\
& \leq \delta_{\mu(u)-1}\left(k b_{k}\left(a_{\mu(u)-1}, a_{\omega(u)}\right)+k b_{k}\left(a_{\omega(u)}, a_{\omega(u)+1}\right)\right) \\
&+\left(1-\delta_{\mu(u)-1}\right)\left[\alpha\left(k b_{k}\left(a_{\mu(u)-1}, a_{\omega(u)}\right)+k b_{k}\left(a_{\omega(u)}, a_{\omega(u)+1}\right)\right)+\beta\left\{b_{k}\left(a_{\mu(u)-1}, f a_{\mu(u)-1}\right)+b_{k}\left(f a_{\omega(u)+1}, a_{\mu(u)+1}\right)\right\}\right. \\
&+\gamma\left\{k b_{k}\left(a_{\mu(u)-1}, a_{\omega(u)}\right)+k^{2} b_{k}\left(a_{\omega(u)}, a_{\omega(u)+1}\right)+k^{2} b_{k}\left(a_{\omega(u)+1}, f a_{\omega(u)+1}\right) k b_{k}\left(a_{\omega(u)}, a_{\omega(u)+1}\right)\right. \\
&\left.\left.+k^{2} b_{k}\left(a_{\mu(u)-1}, a_{\omega(u)}\right)+k^{2} b_{k}\left(a_{\mu(u)-1}, f a_{\mu(u)-1}\right)\right\}+k b_{k}\left(f a_{\omega(u)+1}, a_{\omega(u)+1}\right)\right] .
\end{aligned}
$$


Exerting limit $u \longrightarrow \infty$, we obtain

$$
\begin{aligned}
\lim _{u \longrightarrow \infty} & \sup _{k}\left(a_{\mu(u)}, a_{\omega(u)+1}\right) \\
\leq & \lim _{u \longrightarrow \infty} \sup \left[\delta_{\mu(u)-1}\left(k b_{k}\left(a_{\mu(u)-1}, a_{\omega(u)}\right)\right)+\left(1-\delta_{\mu(u)-1}\right)\left\{\alpha\left(k b_{k}\left(a_{\mu(u)-1}, a_{\omega(u)}\right)\right)\right.\right. \\
& \left.\left.+\gamma\left(k b_{k}\left(a_{\mu(u)-1}, a_{\omega(u)}\right)+k^{2} b_{k}\left(a_{\mu(u)-1}, a_{\omega(u)}\right)\right)\right\}\right] \\
\leq & \lim _{u \longrightarrow \infty} \sup \left[\left(\delta_{\mu(u)-1} k+\left(1-\delta_{\mu(u)-1}\right)\left(\alpha+2 k^{2} \gamma\right)\right) b_{k}\left(a_{\mu(u)-1}, a_{\omega(u)}\right)\right] \\
& <\left(\delta_{\mu(u)-1} k+\left(1-\delta_{\mu(u)-1}\right)\left(\alpha+2 k^{2} \gamma\right)\right) \sigma_{0} \\
\leq & \left(\delta_{\mu(u)-1} k+\left(\alpha+2 k^{2} \gamma\right)\right) \sigma_{0},
\end{aligned}
$$

and hence, by hypothesis, we get

$$
\lim _{u \longrightarrow \infty} \sup _{k}\left(a_{\mu(u)}, a_{\omega(u)+1}\right)<\frac{\sigma_{0}}{k} .
$$

This shows that

$$
\frac{\sigma_{0}}{k} \leq \lim _{u \longrightarrow \infty} \sup b_{k}\left(a_{\mu(u)}, a_{\omega(u)+1}\right)<\frac{\sigma_{0}}{k},
$$

which is a contradiction. Hence, $\left\{a_{n}\right\}$ is a Cauchy sequence. The completeness of $E$ assures the existence of an element $a^{*}$ in $E$ such that

$$
\lim _{n \longrightarrow \infty} b_{k}\left(a_{n}, a^{*}\right)=0 .
$$

However, this is not clear whether the convergent point $a^{*}$ lies inside the closed ball $S_{\varepsilon}\left[a_{0}\right]$ or not. Therefore, before proceeding to the proof of fixed point, we first prove that $a^{*} \in S_{\varepsilon}\left[a_{0}\right]$. By triangular inequality, we write

$$
\begin{aligned}
b_{k} & \left(a_{0}, a^{*}\right) \leq k b_{k}\left(a_{0}, a_{n}\right)+k b_{k}\left(a_{n}, a^{*}\right) \\
& \leq k b_{k}\left(a_{n}, a^{*}\right)+k^{2} b_{k}\left(a_{0}, a_{1}\right)+k^{3} b_{k}\left(a_{1}, a_{2}\right)+\cdots+k^{n+1} b_{k}\left(a_{n-1}, a_{n}\right) \\
& <k b_{k}\left(a_{n}, a^{*}\right)+k^{2}\left[b_{k}\left(a_{0}, a_{1}\right)+k \lambda b_{k} b_{k}\left(a_{0}, a_{1}\right)+\cdots+(k \lambda)^{n-1} b_{k}\left(a_{0}, a_{1}\right)\right] \\
& =k b_{k}\left(a_{n}, a^{*}\right)+k^{2}\left(\frac{1-(k \lambda)^{n-1}}{1-k \lambda}\right) b_{k}\left(a_{0}, a_{1}\right) \\
& \leq k b_{k}\left(a_{n}, a^{*}\right)+k^{2}\left(\frac{1-(k \lambda)^{n-1}}{1-k \lambda}\right) \lambda(1-k \lambda) \mathcal{E},
\end{aligned}
$$

exerting limit $n \longrightarrow \infty$, we get $b_{k}\left(a_{0}, a^{*}\right)<\varepsilon$. That is, $a^{*} \in S_{\varepsilon}\left[a_{0}\right]$. Next, we prove that $a^{*}$ is the fixed point of $f$ :

$$
b_{k}\left(a_{n}, f a^{*}\right) \leq k\left\{b_{k}\left(a_{n}, f a_{n}\right)+b_{k}\left(f a_{n}, f a^{*}\right)\right\} .
$$

$$
F\left(k b_{k}\left(f a_{n}, f a^{*}\right)\right) \leq F\left[\alpha b_{k}\left(a^{*}, a_{n}\right)+\beta\left\{b_{k}\left(a_{n}, f a_{n}\right)+b_{k}\left(a^{*}, f a^{*}\right)\right\}+\gamma\left\{b_{k}\left(a_{n}, f a^{*}\right)+b_{k}\left(a^{*}, f a_{n}\right)\right\}\right]-\tau,
$$

therefore, using above equation and (29),

$$
\begin{aligned}
F\left(b_{k}\left(a_{n}, f a^{*}\right)\right) \leq & F\left[k b_{k}\left(a_{n}, f a_{n}\right)+\alpha b_{k}\left(a^{*}, a_{n}\right)+\beta\left\{b_{k}\left(a_{n}, f a_{n}\right)+b_{k}\left(a^{*}, f a^{*}\right)\right\}+\gamma\left\{b_{k}\left(a_{n}, f a^{*}\right)+b_{k}\left(a^{*}, f a_{n}\right)\right\}\right]-\tau \\
\leq & F\left[k b_{k}\left(a_{n}, f a_{n}\right)+\alpha b_{k}\left(a^{*}, a_{n}\right)+\beta\left\{b_{k}\left(a_{n}, f a_{n}\right)+b_{k}\left(a^{*}, f a^{*}\right)\right\}\right. \\
& \left.+\gamma\left\{k b_{k}\left(a_{n}, a^{*}\right)+k b_{k}\left(a^{*}, f a^{*}\right)+k b_{k}\left(a^{*}, a_{n}\right)+k b_{k}\left(a_{n}, f a_{n}\right)\right\}\right]-\tau
\end{aligned}
$$


and utilizing (F1), we obtain

$$
\begin{aligned}
& F\left(b_{k}\left(a_{n}, f a^{*}\right)-(\beta+k \gamma) b_{k}\left(a^{*}, f a^{*}\right)\right) \\
& \quad \leq F\left[(\alpha+2 \gamma k) b_{k}\left(a^{*}, a_{n}\right)+(k+\beta+k \gamma) b_{k}\left(a_{n}, f a_{n}\right)\right]-\tau \\
& \quad \leq F\left[(\alpha+2 \gamma k) b_{k}\left(a^{*}, a_{n}\right)+(k+\beta+k \gamma) \lambda^{n} b_{k}\left(a_{0}, f a_{0}\right)\right]-\tau .
\end{aligned}
$$

Clearly,

$\lim _{n \longrightarrow \infty}\left((\alpha+2 \gamma k) b_{k}\left(a^{*}, a_{n}\right)+(k+\beta+k \gamma) \lambda^{n} b_{k}\left(a_{0}, f a_{0}\right)\right)=0$, and therefore, by (F2), we get

$$
\lim _{n \longrightarrow \infty} F\left(b_{k}\left(a_{n}, f a^{*}\right)-(\beta+k \gamma) b_{k}\left(a^{*}, f a^{*}\right)\right)=-\infty,
$$

and consequently, by (F2) again, we obtain

$$
\lim _{n \longrightarrow \infty}\left(b_{k}\left(a_{n}, f a^{*}\right)-(\beta+k \gamma) b_{k}\left(a^{*}, f a^{*}\right)\right)=0,
$$

that is, $(1-\beta-k \gamma) b_{k}\left(a^{*}, f a^{*}\right)=0$, Hence $a^{*}$ is the fixed point of $f$. It remains to prove that $a^{*}$ is the only fixed point $f$. Suppose on the contrary that $a^{* *}$ be another fixed point of $f$. Then

$$
\begin{aligned}
F\left(k b_{k}\left(a^{*}, a^{* *}\right)\right) & =F\left(k b_{k}\left(f a^{*}, f a^{* *}\right)\right) \\
& \leq F\left[\alpha b_{k}\left(a^{*}, a^{* *}\right)+\beta\left\{b_{k}\left(a^{*}, f a^{*}\right)+b_{k}\left(a^{* *}, f a^{* *}\right)\right\}+\gamma\left\{b_{k}\left(a^{*}, f a^{* *}\right)+b_{k}\left(a^{* *}, f a^{*}\right)\right\}\right]-\tau \\
& =F\left[(\alpha+2 \gamma) b_{k}\left(a^{*}, a^{* *}\right)\right]-\tau
\end{aligned}
$$

which is a contradiction because $k \geq 1, F$ is strictly increasing, and by hypothesis, $\alpha+2 \gamma<1$. Hence, $b_{k}\left(a^{*}, a^{* *}\right)=0$. That is, $a^{*}=a^{* *}$.

Example 1. Suppose $E=[0,+\infty)$ and $\varepsilon=a_{0}=(1 / 2)$, then $S_{\varepsilon}[1 / 2]=I$. Let $f: E \longrightarrow E$ be defined by

$$
f a= \begin{cases}e^{(-3 / 4)}, & \text { if } a=\frac{1}{2} \\ \frac{a}{6}, & \text { otherwise }\end{cases}
$$

and choose a mapping $b_{k}: E \times E \longrightarrow[0,+\infty)$ defined as

$$
b_{k}(a, b)= \begin{cases}(x-y)^{2}, & \text { if both } x, y \in[0,1] \\ |x-y|, & \text { otherwise. }\end{cases}
$$

Demonstrate $\quad \eta: E \times E \times I \longrightarrow E \quad$ as $\quad \eta(a, b ; \delta)=$ $\delta a+(1-\delta) b$ for all $a, b \in E$. Choose $a_{n}=\eta\left(a_{n-1}, f a_{n-1}\right.$; $\left.\delta_{n-1}\right), k=2$, and fix $\delta_{n-1}=(1 / 16)=\left(1 / 4 k^{2}\right)$. Observe that $\delta a+(1-\delta) b \in[0,1]$ whenever $a, b \in[0,1]$. Now, consider $o, a, b \in E$, then we have two cases:

(i) When $o \notin[0,1]$, we get

$$
\begin{aligned}
b_{k}(o, \eta(a, b ; \delta)) & =|\delta(o-a)+(1-\delta)(o-b)| \\
& \leq|\delta(o-a)|+|(1-\delta)(o-b)| \\
& =\delta b_{k}(o, a)+(1-\delta) b_{k}(o, b)
\end{aligned}
$$

(ii) When $o \in[0,1]$. We have the following subcases:

(a) If both $a, b \in[0,1]$, then obviously $\eta(a, b ; \delta)=$ $\delta a+(1-\delta) b \in[0,1]$, and hence

$$
\begin{aligned}
b_{k}(o, \eta(a, b, \gamma))= & {[\gamma(o-a)+(1-\gamma)(o-b)]^{2} } \\
\leq & {[\gamma|o-a|+(1-\gamma)|o-b|]^{2} } \\
= & (\gamma|o-a|)^{2}+((1-\gamma)|o-b|)^{2} \\
& +2 \gamma(1-\gamma)|o-a||o-b| \\
\leq & (\gamma|o-a|)^{2}+((1-\gamma)|o-b|)^{2} \\
& +\gamma(1-\gamma)\left((o-a)^{2}+(o-b)^{2}\right) \\
= & \gamma(o-a)^{2}+(1-\gamma)(o-b)^{2} \\
= & \gamma b_{k}(o, a)+(1-\gamma) b_{k}(o, b) .
\end{aligned}
$$

(b) If only one of $a$ and $b$ is in $[0,1]$, say $a$ is in $[0,1]$, then obviously $\eta(a, b ; \delta)=\delta a+(1-\delta) b \notin$ $[0,1]$, and hence,

$$
\begin{aligned}
b_{k}(o, \eta(a, b ; \delta)) & =|\delta(o-a)+(1-\delta)(o-b)| \\
& \leq|\delta(o-a)|+|(1-\delta)(o-b)| \\
& \leq \delta(o-a)^{2}+|(1-\delta)(o-b)| \\
& =\delta b_{k}(o, a)+(1-\delta) b_{k}(o, b) .
\end{aligned}
$$

The same can be done for $b$ in $[0,1]$ and $a$ not in $[0,1]$.

(c) If both $a, b \notin[0,1]$, then obviously $\eta(a, b ; \delta)=\delta a+(1-\delta) b \notin[0,1]$, and hence

$$
\begin{aligned}
b_{k}(o,(a, b ; \delta)) & =|\delta(o-a)+(1-\delta)(o-b)| \\
\leq & |\delta(o-a)|+|(1-\delta)(o-b)| \\
& =|\delta(o-a)|+|(1-\delta)(o-b)| \\
& =\delta b_{k}(o, a)+(1-\delta) b_{k}(o, b) .
\end{aligned}
$$


From all the possible cases, it is clear that $\left(E, b_{k}, \eta\right)$ is a convex b-metric space with $k=2$. Next, fix $\beta=\gamma=0$ and $\alpha=(1 / 16)$. Observe that

$$
k\left(k^{2} \delta_{n-1}+\alpha k\right)+\left(k^{2}+1\right)(\beta+2 \gamma k)=k\left(k^{2} \frac{1}{4 k^{2}}+\alpha k\right)<1 .
$$

Also

$$
\begin{aligned}
\lambda(1-k \lambda) \mathcal{E}= & \frac{\left(k^{2} \delta_{m-1}+\alpha+\beta+k \gamma+\gamma \delta_{m-1}\right)}{(1-\beta-k \gamma)} \\
& \cdot\left(1-k \frac{\left(k^{2} \delta_{m-1}+\alpha+\beta+k \gamma+\gamma \delta_{m-1}\right)}{(1-\beta-k \gamma)}\right) \frac{1}{2} \\
= & \left(k^{2} \delta_{m-1}+\alpha\right)\left(1-k\left(k^{2} \delta_{m-1}+\alpha\right)\right) \frac{1}{2} \\
& =\left(\frac{5}{16}\right)\left(1-\frac{5}{8}\right) \frac{1}{2}>0.00076 \\
& \cong b_{k}\left(\frac{1}{2}, e^{(-3 / 4)}\right) \\
& =b_{k}\left(a_{0}, f a_{0}\right),
\end{aligned}
$$

that is, $b_{k}\left(a_{0}, f a_{0}\right)<\lambda(1-k \lambda) \varepsilon$. Now checking for the contractive condition, we again have two cases:

Case 1: if both $a, b \in[0,1]$, then

$$
\begin{aligned}
\ln \left(k b_{k}(f a, f b)\right)= & \ln \left\{k\left(\frac{a}{6}-\frac{b}{6}\right)^{2}\right\} \\
= & \ln \left(k \frac{1}{36}(a-b)^{2}\right) \leq \ln \left[\alpha(a-b)^{2}\right] \\
= & \ln \left[\alpha b_{k}(a, b)+\beta\left\{b_{k}(a, f a)+b_{k}(b, f b)\right\}\right. \\
& \left.+\gamma\left\{b_{k}(a, f b)+b_{k}(b, f a)\right\}\right],
\end{aligned}
$$

that is,

$$
\begin{aligned}
F\left(k b_{k}(f a, f b)\right) \leq & F\left[\alpha b_{k}(a, b)+\beta\left\{b_{k}(a, f a)+b_{k}(b, f b)\right\}\right. \\
& \left.+\gamma\left\{b_{k}(a, f b)+b_{k}(b, f a)\right\}\right] .
\end{aligned}
$$

Thus, the inequality (4) is satisfied for $\tau \in\left(0, \ln \left(\left(4 k^{2}+\right.\right.\right.$ $\left.\left.2) /\left(4 k^{2}+1\right)\right)\right)$ and for all $a, b \in[0,1]$.

Case 2: if both $a, b \notin[0,1]$, i.e., say $a=12$ and $b=6$, then

$$
\begin{aligned}
\ln \left(k b_{k}(f a, f b)\right)= & \ln \{k|2-1|\}>\ln \left(\frac{1}{16}|12-6|\right) \\
= & \ln \left[\alpha b_{k}(a, b)\right] \\
= & \ln \left[\alpha b_{k}(a, b)+\beta\left\{b_{k}(a, f a)+b_{k}(b, f b)\right\}\right. \\
& \left.+\gamma\left\{b_{k}(a, f b)+b_{k}(b, f a)\right\}\right] .
\end{aligned}
$$

Hence the inequality (4) does not hold for all the elements of $E$. Hence the contractive condition (4) holds true only for $a, b \in[0,1]$. Observe that $F(x)=\ln (x)$ satisfies (F1) and (F2). Moreover

$$
\begin{aligned}
a_{n} & =\delta_{n-1} a_{n-1}+\left(1-\delta_{n-1}\right) f a_{n-1} \\
& =\delta_{n-1} a_{n-1}+\left(1-\delta_{n-1}\right) \frac{a_{n-1}}{6} \\
& =\left(\frac{5}{6} \delta_{n-1}+\frac{1}{6}\right) a_{n-1}=\frac{17}{96} a_{n-1} .
\end{aligned}
$$

Similarly

$$
a_{n-1}=\frac{17}{96} a_{n-2}, a_{n-2}=\frac{17}{96} a_{n-3}, \ldots, a_{1}=\frac{17}{96} a_{0} .
$$

Therefore, $a_{n}=(17 / 96)^{n} a_{0}$ and $f a_{n}=(1 / 6)(17 / 96)^{n} a_{0}$. Letting $n \longrightarrow \infty$, we get $a_{n} \longrightarrow 0$ and $f a_{n} \longrightarrow 0$. That is, 0 is the fixed point of $f$. For uniqueness, suppose on contrary that $r$ is another fixed point of $f$ then $b_{k}(0, r)>0$, say $b_{k}(0, r)=\delta$. Hence

$$
\begin{aligned}
\delta & =b_{k}(0, r)=b_{k}(f 0, f r)=\left(0-\frac{r}{5}\right)^{2}=\frac{r^{2}}{25} \\
& =\frac{1}{25} b_{k}(0, r)=\frac{1}{25} \delta,
\end{aligned}
$$

which is a contradiction. Therefore, 0 is the only fixed point of $f$.

Taking $\beta=0$ in Theorem 1 , we obtain the following results.

Corollary 1. Assume that $F \in \mathscr{F}_{b},\left(E, b_{k}, \eta\right)$ is a complete convex b-metric space with $k>1$ and $\left\{a_{n}\right\}$ is a generalized Mann's iteration having $\delta_{n-1} \in\left(0,\left(1 / 4 k^{2}\right)\right]$ for all $n \in N$. Suppose $S_{\varepsilon}\left[a_{0}\right]$ be a closed ball in $E$ and for $\alpha, \gamma \in[0, \infty)$ with $k\left(k^{2} \delta_{n-1}+\alpha k\right)+\left(k^{2}+1\right) 2 \gamma k<1, \lambda=\left(\left(k \delta_{n-1}+\alpha+k \gamma+\gamma \delta_{n-1}\right) /\right.$ $(1-k \gamma))$. If $f: E \longrightarrow E$ is a mapping such that for $a, b \in[0,1]$, the following holds:

$$
\begin{aligned}
\tau+F\left(k b_{k}(f a, f b)\right) & \leq F\left[\alpha b_{k}(a, b)+\gamma\left\{b_{k}(a, f b)+b_{k}(b, f a)\right\}\right] ; \\
b_{k}\left(a_{0}, f a_{0}\right) & \leq \lambda(1-k \lambda) \mathcal{\varepsilon} .
\end{aligned}
$$

Then $f$ has a unique fixed point in $E$.

Taking $\gamma=0$ in Theorem 1 , we obtain the following results.

Corollary 2. Assume that $F \in \mathscr{F}_{b},\left(E, b_{k}, \eta\right)$ is a complete convex $b$-metric space with $k>1$ and $\left\{a_{n}\right\}$ is a generalized Mann's iteration having $\delta_{n-1} \in\left(0,\left(1 / 4 k^{2}\right)\right]$ for all $n \in N$. Suppose $S_{\varepsilon}\left[a_{0}\right]$ be a closed ball in $E$ and for $\alpha, \beta \in[0, \infty)$ with $k\left(k^{2} \delta_{n-1}+\alpha k\right)+\left(k^{2}+1\right) \beta<1, \lambda=\left(\left(k \delta_{n-1}+\alpha+\beta\right) /(1-\beta)\right)$. If $f: E \longrightarrow E$ is a mapping such that for $a, b \in[0,1]$, the following holds: 
$\tau+F\left(k b_{k}(f a, f b)\right) \leq F\left[\alpha b_{k}(a, b)+\beta\left\{b_{k}(a, f a)+b_{k}(b, f b)\right\}\right] ;$ $b_{k}\left(a_{0}, f a_{0}\right) \leq \lambda(1-k \lambda) \varepsilon$.

Then $f$ has a unique fixed point in $E$.

\section{Fixed Point Results of Globally Contractive Mappings $f$ on Convex $\boldsymbol{b}$-Metric Space}

In this section, fixed point is instigated for $F$-contraction of Hardy-Roger's type while the contractive condition holds true on the whole b-metric space rather than locally only on closed ball.

Theorem 2. Suppose $F \in \mathscr{F}_{b},\left(E, b_{k}, \eta\right)$ is a complete convex $b$-metric space with $k>1$ and $\left\{a_{n}\right\}$ is a generalized Mann's iteration having $\delta_{n-1} \in\left(0,\left(1 / 4 k^{2}\right)\right]$ for all $n \in N$. If for $\alpha, \beta, \gamma \in[0, \infty) \quad$ with $\quad k\left(k \delta_{n-1}+\alpha\right)+\left(k+\delta_{n-1}+1\right)$ $(\beta+\gamma k)<1$ and $f: E \longrightarrow E$ such that the following hold:

$$
\begin{aligned}
\tau+F\left(k b_{k}(f a, f b)\right) \leq & F\left[\alpha b_{k}(a, b)+\beta\left\{b_{k}(a, f a)+b_{k}(b, f b)\right\}\right. \\
+ & \left.\gamma\left\{b_{k}(a, f b)+b_{k}(b, f a)\right\}\right],
\end{aligned}
$$

for every $a, b \in E$. Then $f$ has a unique fixed point in $E$.

Proof. We know by hypothesis

$$
\begin{aligned}
& b_{k}\left(a_{m}, a_{m+1}\right) \leq\left(1-\delta_{m}\right) b_{k}\left(a_{m}, f a_{m}\right), \\
& b_{k}\left(a_{m}, f a_{m}\right) \leq k b_{k}\left(a_{m}, f a_{m-1}\right)+k b_{k}\left(f a_{m-1}, f a_{m}\right) .
\end{aligned}
$$

By (53),

$$
\begin{gathered}
\tau+F\left(k b_{k}\left(f a_{m-1}, f a_{m}\right)\right) \leq \\
F\left[\alpha b_{k}\left(a_{m-1}, a_{m}\right)+\beta\left\{b_{k}\left(a_{m-1}, f a_{m-1}\right)+b_{k}\left(a_{m}, f a_{m}\right)\right\}\right. \\
\left.+\gamma\left\{b_{k}\left(a_{m-1}, f a_{m}\right)+b_{k}\left(a_{m}, f a_{m-1}\right)\right\}\right]
\end{gathered}
$$

and this implies that

$$
\begin{aligned}
F\left(b_{k}\left(a_{m}, f a_{m}\right)\right)= & F\left(k b_{k}\left(a_{m}, f a_{m-1}\right)+k b_{k}\left(f a_{m-1}, f a_{m}\right)\right) \\
\leq & F\left[k b_{k}\left(a_{m}, f a_{m-1}\right)+\alpha\left(1-\delta_{m-1}\right) b_{k}\left(a_{m-1}, f a_{m-1}\right)+\beta\left\{\begin{array}{c}
b_{k}\left(a_{m-1}, f a_{m-1}\right) \\
+b_{k}\left(a_{m}, f a_{m}\right)
\end{array}\right\}\right. \\
& \left.+\gamma\left\{b_{k}\left(a_{m-1}, f a_{m}\right)+b_{k}\left(a_{m}, f a_{m-1}\right)\right\}\right]-\tau \\
\leq & F\left[k \delta_{m-1} b_{k}\left(a_{m-1}, f a_{m-1}\right)+\alpha\left(1-\delta_{m-1}\right) b_{k}\left(a_{m-1}, f a_{m-1}\right)\right. \\
& +\beta\left\{b_{k}\left(a_{m-1}, f a_{m-1}\right)+b_{k}\left(a_{m}, f a_{m}\right)\right\}+\gamma\left\{k b_{k}\left(a_{m-1}, a_{m}\right)+k b_{k}\left(a_{m}, f a_{m}\right)+\delta_{m-1} b_{k}\left(a_{m-1}, f a_{m-1}\right)\right\}-\tau \leq \\
\leq & F k \delta_{m-1} b_{k}\left(a_{m-1}, f a_{m-1}\right)+\alpha\left(1-\delta_{m-1}\right) b_{k}\left(a_{m-1}, f a_{m-1}\right) \\
& +\beta\left\{b_{k}\left(a_{m-1}, f a_{m-1}\right)+b_{k}\left(a_{m}, f a_{m}\right)\right\}+\gamma\left\{k\left(1-\delta_{m-1}\right) b_{k}\left(a_{m-1}, f a_{m-1}\right)\right. \\
& \left.+k b_{k}\left(a_{m}, f a_{m}\right)+\delta_{m-1} b_{k}\left(a_{m-1}, f a_{m-1}\right)\right\}-\tau,
\end{aligned}
$$

using (F1), we write

$$
\begin{aligned}
(1-\beta-k \gamma) b_{k}\left(a_{m}, f a_{m}\right) \leq & \left(k \delta_{m-1}+\alpha\left(1-\delta_{m-1}\right)+\beta\right. \\
+ & \left.k\left(1-\delta_{m-1}\right) \gamma+\gamma \delta_{m-1}\right) \\
& \cdot b_{k}\left(a_{m-1}, f a_{m-1}\right),
\end{aligned}
$$

and this implies that

$$
\begin{aligned}
b_{k}\left(a_{m}, f a_{m}\right) & <\frac{\left(k \delta_{m-1}+\alpha+\beta+k \gamma+\gamma \delta_{m-1}\right)}{(1-\beta-k \gamma)} b_{k}\left(a_{m-1}, f a_{m-1}\right) \\
& =\lambda b_{k}\left(a_{m-1}, f a_{m-1}\right),
\end{aligned}
$$

where $\lambda=\left(\left(k \delta_{m-1}+\alpha+\beta+k \gamma+\gamma \delta_{m-1}\right) /(1-\beta-k \gamma)\right)$. Or we can say that for all $m \in N$,

$$
b_{k}\left(a_{m}, f a_{m}\right)<\lambda b_{k}\left(a_{m-1}, f a_{m-1}\right) .
$$

Note that $\lambda<1$. Now from (53), (54), and (59), we write

$$
\begin{aligned}
F\left(b_{k}\left(a_{n}, a_{n+1}\right)\right) & \leq F\left(b_{k}\left(a_{n-1}, f a_{n-1}\right)\right)-\tau \\
& \leq F\left(b_{k}\left(a_{n-2}, f a_{n-2}\right)\right)-2 \tau \leq \cdots \\
& \leq F\left(b_{k}\left(a_{0}, f a_{0}\right)\right)-n \tau,
\end{aligned}
$$

exerting limit $n \longrightarrow \infty$, we obtain

$$
\lim _{n \longrightarrow \infty} F\left(b_{k}\left(a_{n}, f a_{n}\right)\right)=-\infty,
$$

consequently by (F2), we acquire 


$$
\lim _{n \longrightarrow \infty} b_{k}\left(a_{n}, f a_{n}\right)=0 .
$$

Hence,

$$
\lim _{n \longrightarrow \infty} b_{k}\left(a_{n}, a_{n+1}\right)=\lim _{n \longrightarrow \infty} b_{k}\left(a_{n}, f a_{n}\right)=0 .
$$

The Cauchyness of the sequence $\left\{a_{n}\right\}$ can be proved by following the same steps as in equation (59). Afterwards, on contrary to the previous of the theorem, we do not need to prove convergence as every Cauchy sequence in complete b-metric space is Convergent. Hence there exist an element $a^{*}$ in $E$ such that $\lim _{n \longrightarrow \infty} a_{n} \longrightarrow a^{*}$. Furthermore, $a^{*}$ can be proved as the unique fixed point of $f$ in the similar way as done in Theorem 1.

Corollary 3. Suppose $F \in \mathscr{F}_{b},\left(E, b_{k}, \eta\right)$ is a complete convex $b$-metric space with $k>1$ and $\left\{a_{n}\right\}$ is a generalized Mann's iteration having $\delta_{n-1} \in\left(0,\left(1 / 4 k^{2}\right)\right]$ for all $n \in N$. If for $\alpha, \beta \in[0, \infty)$ with $k\left(k \delta_{n-1}+\alpha k\right)+\left(k^{2}+1\right) \beta<1 \quad$ and $f: E \longrightarrow E$, such that the following hold:

$$
\tau+F\left(k b_{k}(f a, f b)\right) \leq F\left[\alpha b_{k}(a, b)+\beta\left\{b_{k}(a, f a)+b_{k}(b, f b)\right\}\right],
$$

or every $a, b \in E$. Then $f$ has a unique fixed point in $E$.

\section{Application}

In Physics and Engineering, many problems are modelled in to second-order linear differential equations, i.e., system of an object with mass attached to a vertical spring, an inductor, a capacitor connected in series, and an electric circuit with a resistor. The differential equation discussed in this section shows the engineering problem of activation of spring affected by external forces. The differential equation will be reformulated to fixed point problems and the existence of the fixed point will assure the existence of the solution of differential equation (see $[1,17]$ ).

Suppose the boundary value problem for second-order differential equation be

$$
\left\{\begin{array}{l}
\frac{\mathrm{d}^{2} a}{\mathrm{~d} s^{2}}+\frac{c}{n} \frac{\mathrm{d} a}{\mathrm{~d} s}=A(s, a(s)), \\
a(0)=0, a^{\prime}(0)=l,
\end{array}\right.
$$

where $A: I \times R^{+} \longrightarrow R$ is a continuous function. Equation (65) can be equivalently written as

$$
a(s)=\int_{0}^{s} B(s, r) A(r, a(r)) \mathrm{d} r, \quad s \in I,
$$

where $B(s, r)$ is the green function stated as

$$
B(s, r)= \begin{cases}(s-r) e^{\tau(s-r)}, & \text { if } 0 \leq r \leq S \leq 1, \\ 0, & \text { if } 0 \leq s \leq r \leq 1\end{cases}
$$

With $\tau>0$ formulated in terms of $m$ and $c$ in (66). Suppose $E:=C\left([0,1], R^{+}\right)$is the collection of all continuous functions from $[0,1]$ into $R^{+}$. Define

$$
\|a\|_{\tau}=\operatorname{Sup}_{s \in I}\left(|a(s)| e^{-2 \tau s}\right),
$$

for $a \in E$, and $b_{k}: E \times E \longrightarrow R^{+}$as

$$
b_{k}(f, g)=\left(\|f\|_{\tau}-\|g\|_{\tau}\right)^{2} .
$$

Consider $T: E \longrightarrow E$ is defined by

$$
T(a(s))=\int_{0}^{s} B(s, r) A(r, a(r)) \mathrm{d} r
$$

for all $a \in E$ and $s \in I$. Note that the existence of fixed point of $T$ guarantees the existence of solution of equation (66).

Before starting our main theorem, we first prove the following important lemma.

Lemma 1. Suppose $E:=C\left([0,1], R^{+}\right), b_{k}$ is defined by (69) and $\eta: E \times E \times\left(0,\left(1 / 4 k^{2}\right)\right]$ is stated as $\eta(f, g, \delta)(p)=\delta f(p)+(1-\delta) g(p)$ for all $p \in I$, then $\left(E, b_{k}, \eta\right)$ is a convex $b$-metric space.

Proof. For $f, g, h \in E, \tau>0$, and $\delta \in[0,1]$,

$$
\begin{aligned}
b_{k}(f, \eta(g, h, \delta)) & =b_{k}\left(\|f\|_{\tau}-\|\delta g+(1-\delta) h\|_{\tau}\right)^{2} \leq b_{k}\left(\left(\|f\|_{\tau}-|\delta|\|g\|_{\tau}-|1-\delta|\|h\|_{\tau}\right)\right)^{2} \\
& =\left[\delta\left(\|f\|_{\tau}-\|g\|_{\tau}\right)+(1-\delta)\left(\|f\|_{\tau}-\|h\|_{\tau}\right)\right]^{2} \\
& \leq\left[\delta\left(\left|\|f\|_{\tau}-\|g\|_{\tau}\right|\right)+(1-\delta)\left(\left|\|f\|_{\tau}-\|h\|_{\tau}\right|\right)\right]^{2} \\
& =\left(\delta\left|\|f\|_{\tau}-\|g\|_{\tau}\right|\right)^{2}+\left((1-\delta)\left|\|f\|_{\tau}-\|h\|_{\tau}\right|\right)^{2}+2 \delta(1-\delta)\left(\left|\|f\|_{\tau}-\|g\|_{\tau}\right|\right)\left(\left|\|f\|_{\tau}-\|h\|_{\tau}\right|\right) \\
& \leq\left(\delta\left|\|f\|_{\tau}-\|g\|_{\tau}\right|\right)^{2}+\left((1-\delta)\left|\|f\|_{\tau}-\|h\|_{\tau}\right|\right)^{2}+\delta(1-\delta)\left(\left(\left|\|f\|_{\tau}-\|g\|_{\tau}\right|\right)^{2}+\left(\|f\|_{\tau}-\|h\|_{\tau}\right)^{2}\right) \\
& =\delta\left(\|f\|_{\tau}-\|g\|_{\tau}\right)^{2}+(1-\delta)\left(\|f\|_{\tau}-\|h\|_{\tau}\right)^{2}=\delta b_{k}(f, g)+(1-\delta) b_{k}(f, h) .
\end{aligned}
$$

Hence $b_{k}$ is convex b-metric space with $k=2$. 
Theorem 3. Suppose the integral equation (66) and let the following condition holds:

(1) $A$ is an increasing function.

(2) There exist $\tau>0$ such as

$$
|A(r, a)| \leq \frac{\lambda \tau^{2} e^{-\tau}}{\sqrt{k}} a
$$

where $\lambda \in[0,1), r \in I$, and $a(r) \in R^{+}$.

(3) For $\alpha, \beta, \gamma \in[0, \infty)$ with $k\left(k \delta_{n-1}+\alpha\right)+\left(k+\delta_{n-1}+\right.$ 1) $(\beta+\gamma k)<1$ and the norm $\|\cdot\|_{\tau}$ defined above, the following hold:

$$
\left(\lambda e^{-\tau}\right)^{2}\left(\|a\|_{\tau}-\|b\|_{\tau}\right)^{2} \leq \alpha\left(\|a\|_{\tau}-\|b\|_{\tau}\right)^{2}+\left\{\left(\beta\left(1-\frac{\lambda e^{-\tau}}{\sqrt{k}}\right)^{2}+\gamma\left(1+\frac{\left(\lambda e^{-\tau}\right)^{2}}{k}\right)\right) \cdot\left(\|a\|_{\tau}^{2}+\|b\|_{\tau}^{2}\right)\right\}-4 \gamma \frac{\lambda e^{-\tau}}{\sqrt{k}}\|a\|_{\tau}\|b\|_{\tau}
$$

for $\delta_{n-1} \in\left(0,\left(1 / 4 k^{2}\right)\right]$. Then the equation (69) has a solution.

Proof. By (70),

$$
\begin{aligned}
|T(a(r))| & =\left|\int_{0}^{s} B(s, r) A(r, a(r)) \mathrm{d} r\right| \\
& \leq \int_{0}^{s} B(s, r)|A(r, a(r))| \mathrm{d} r \\
& \leq \int_{0}^{s} B(s, r) \frac{\lambda \tau^{2} e^{-\tau}}{\sqrt{k}}|a(r)| \mathrm{d} r \\
& =\int_{0}^{s} B(s, r) \frac{\lambda \tau^{2} e^{-\tau}}{\sqrt{k}} e^{2 \tau r} e^{-2 \tau r}|a(r)| \mathrm{d} r \\
& \leq \int_{0}^{s} \frac{\lambda \tau^{2} e^{-\tau}}{\sqrt{k}}(s-r) e^{\tau(s-r)} e^{2 \tau r}\|a\|_{\tau} \mathrm{d} r \\
& =\frac{\lambda \tau^{2}}{\sqrt{k}} e^{-\tau+\tau s}\|a\|_{\tau} \int_{0}^{s}(s-r) e^{\tau r} \mathrm{~d} r \\
& =\frac{\lambda \tau^{2}}{\sqrt{k}} e^{-\tau+\tau s}\|a\|_{\tau}\left[\frac{-s}{\tau}-\frac{1}{\tau^{2}}+\frac{e^{\tau s}}{\tau^{2}}\right] \\
& =\frac{\lambda e^{-\tau}}{\sqrt{k}}\|a\|_{\tau} e^{2 \tau s}\left[1-\tau s e^{-\tau s}-e^{-\tau s}\right] . \\
&
\end{aligned}
$$

As $\left[1-\tau s e^{-\tau s}-e^{-\tau s}\right]<1$, then

$$
\|\operatorname{Ta}(r)\|_{\tau} \leq \frac{\lambda e^{-\tau}}{\sqrt{k}}\|a\|_{\tau}
$$

Similarly, we obtain

$$
\|T b(r)\|_{\tau} \leq \frac{\lambda e^{-\tau}}{\sqrt{k}}\|b\|_{\tau}
$$

Now

$$
b_{k}(T a, T b)=\left(\|T a\|_{\tau}-\|T b\|_{\tau}\right)^{2} \leq \frac{\left(\lambda e^{-\tau}\right)^{2}}{k}\left(\|a\|_{\tau}-\|b\|_{\tau}\right)^{2}
$$

or

$$
k b_{k}(T a, T b) \leq\left(\lambda e^{-\tau}\right)^{2}\left(\|a\|_{\tau}-\|b\|_{\tau}\right)^{2}
$$

By hypothesis, we write

$$
\begin{aligned}
k b_{k}(T a, T b) \leq & \alpha\left(\|a\|_{\tau}-\|b\|_{\tau}\right)^{2}+\left(\beta\left(1-\frac{\lambda e^{-\tau}}{\sqrt{k}}\right)^{2}+\gamma\left(1+\frac{\left(\lambda e^{-\tau}\right)^{2}}{k}\right)\right) \cdot\left(\|a\|_{\tau}^{2}-\|b\|_{\tau}^{2}\right)-4 \gamma \frac{\lambda e^{-\tau}}{\sqrt{k}}\|a\|_{\tau}\|b\|_{\tau} \\
= & \alpha\left(\|a\|_{\tau}-\|b\|_{\tau}\right)^{2}+\beta\left\{\left(1-\frac{\lambda e^{-\tau}}{\sqrt{k}}\right)^{2}\left(\|a\|_{\tau}^{2}-\|b\|_{\tau}^{2}\right)\right\} \\
& +\gamma\left\{\left(\|a\|_{\tau}^{2}-\|b\|_{\tau}^{2}\right)\left(1+\frac{\left(\lambda e^{-\tau}\right)^{2}}{k}\right)-4 \frac{\lambda e^{-\tau}}{\sqrt{k}}\|a\|_{\tau}\|b\|_{\tau}\right\}
\end{aligned}
$$




$$
\begin{aligned}
= & \alpha\left(\|a\|_{\tau}-\|b\|_{\tau}\right)^{2}+\beta\left\{\|a\|_{\tau}^{2}\left(1-\frac{\lambda e^{-\tau}}{\sqrt{k}}\right)^{2}+\|b\|_{\tau}^{2}\left(1-\frac{\lambda e^{-\tau}}{\sqrt{k}}\right)^{2}\right\} \\
& +\gamma\left\{\|a\|_{\tau}^{2}+\left(\frac{\lambda e^{-\tau}}{\sqrt{k}}\right)^{2}\|b\|_{\tau}^{2}-2 \frac{\lambda e^{-\tau}}{\sqrt{k}}\|a\|_{\tau}\|b\|_{\tau}+\|b\|_{\tau}^{2}+\left(\frac{\lambda e^{-\tau}}{\sqrt{k}}\right)^{2}\|a\|_{\tau}^{2}-2 \frac{\lambda e^{-\tau}}{\sqrt{k}}\|b\|_{\tau}\|a\|_{\tau}\right\} \\
= & \alpha\left(\|a\|_{\tau}-\|b\|_{\tau}\right)^{2}+\beta\left\{\left(\|a\|_{\tau}-\frac{\lambda e^{-\tau}}{\sqrt{k}}\|a\|_{\tau}\right)^{2}+\left(\|b\|_{\tau}-\frac{\lambda e^{-\tau}}{\sqrt{k}}\|b\|_{\tau}\right)^{2}\right\} \\
& +\gamma\left\{\left(\|a\|_{\tau}-\frac{\lambda e^{-\tau}}{\sqrt{k}}\|b\|_{\tau}\right)^{2}+\left(\|b\|_{\tau}-\frac{\lambda e^{-\tau}}{\sqrt{k}}\|a\|_{\tau}\right)^{2}\right\} \\
= & \alpha b_{k}(a, b)+\beta\left\{b_{k}(a, T a)+b_{k}(b, T b)\right\}+\gamma\left\{b_{k}(a, T a)+b_{k}(b, T b)\right\} .
\end{aligned}
$$

Therefore,

$$
\begin{aligned}
\ln \left(k b_{k}(T a, T b)\right) \leq & \ln \left[\alpha b_{k}(a, b)+\beta\left\{b_{k}(a, T a)+b_{k}(b, T b)\right\}\right. \\
& \left.+\gamma\left\{b_{k}(a, T b)+b_{k}(b, T a)\right\}\right],
\end{aligned}
$$

$$
\begin{aligned}
\tau+F\left(k b_{k}(T a, T b)\right) \leq & F\left[\alpha b_{k}(a, b)+\beta\left\{b_{k}(a, T a)+b_{k}(b, T b)\right\}\right. \\
& \left.+\gamma\left\{b_{k}(a, T b)+b_{k}(b, T a)\right\}\right],
\end{aligned}
$$

and for

and hence,

$$
\tau=(0, \rho), \quad \text { where } \rho=\ln \left(\frac{\alpha b_{k}(a, b)+\beta\left\{b_{k}(a, T a)+b_{k}(b, T b)\right\}+\gamma\left\{b_{k}(a, T b)+b_{k}(b, T a)\right\}}{k b_{k}(T a, T b)}\right)
$$

Therefore, all the conditions of the Theorem 2 are satisfied, so the integral equation (66) has a unique solution. Consequently, the differential equation (65) has a unique solution.

\section{Conclusion}

This paper has introduced F-Hardy-Roger's contraction on closed ball in convex b-metric space, and generalized Mann's iteration theorem algorithm is used to find the fixed point. The existence of the limit point inside the closed ball is established despite of the fact that the subset closed ball in a complete b-metric space is not complete. The convergence of the chosen sequence is insured inside the closed ball without completeness of the ball. Hence, a new approach is used to investigate fixed point for F-HardyRoger's contractions (and can extend similarly to others contractions). The results are followed by an interesting example in $R^{2}$. Furthermore, an application of our results in finding a unique solution to second-order differential equation with boundary valued is described along with an important lemma. The paper furthered the research already done on the topic of $F$-contractions and fixed point theory. In future, these results will be formulated in the structure of Hilbert spaces and orthogonal partial b-metric spaces, and its application in convex minimization and fractional differential equations will be investigated in continuation to the work already done in [21-25].

\section{Data Availability}

No data are used in this research.

\section{Conflicts of Interest}

The authors declare no conflicts of interest.

\section{Authors' Contributions}

All the authors contributed equally to this research work.

\section{Acknowledgments}

This project was funded by the Deanship of Scientific Research (DSR), King Abdulaziz University, Jeddah, Saudi Arabia, under grant no. KEP-66-130-38. The authors, therefore, acknowledge with thanks DSR technical and financial support.

\section{References}

[1] A. A. Branciari, "Fixed point theorem of Banach-Caccioppoli type on a class of generalized metric spaces," Publicationes Mathematicae, vol. 57, pp. 31-37, 2000.

[2] A. Hussain and T. Kanwal, "Existence and uniqueness for a neutral differential problem with unbounded delay via fixed point results," Transactions of A. Razmadze Mathematical Institute, vol. 172, pp. 481-490, 2018. 
[3] M. Jleli and B. Samet, "A generalized metric space and related fixed point theorems," Fixed Point Theory and Applications, vol. 61, p. 14, 2015.

[4] M. Jleli and B. Samet, "On a new generalization of metric spaces," Journal of Fixed Point Theory and Applications, vol. 20, no. 3, p. 128, 2018.

[5] S. Malhotra, S. Shukla, and R. Sen, "Some fixed point theorems for ordered Reich type contractions in cone rectangular metric spaces," Acta Mathematica Universitatis Comenianae, vol. 82, no. 2, pp. 165-175, 2013.

[6] S. G. Matthews, "Partial metric topology," Annals of the New York Academy of Sciences, vol. 728, pp. 183-197, 1994.

[7] Z. Mustafa and B. Sims, "A new approach to generalized metric spaces," Journal of Nonlinear and Convex Analysis, vol. 7, no. 2, pp. 289-297, 2006.

[8] S. Czerwik, "Contraction mappings in $b$-metric spaces," Acta Mathematica et Informatica Universitatis Ostraviensis, vol. 1, no. 1, pp. 5-11, 1993.

[9] M. A. Alghamdi, N. Hussain, and P. Salimi, "Fixed point and coupled fixed point theorems on $b$-metric-like spaces," Journal of Inequalities and Applications, vol. 402, 2013.

[10] S. Shukla, "Partial b-metric spaces and fixed-point theorems," Mediterranean Journal of Mathematics \& Computer Science, vol. 11, pp. 703-714, 2014.

[11] L. Chen, C. Li, R. Kaczmarek, and Y. Zhao, "Several fixed point theorems in convex b-metric spaces and applications," Mathematics, vol. 8, no. 2, p. 242, 2020.

[12] M. Arshad, M. Abbas, A. Hussain, and N. Hussain, "Generalized dynamic process for generalized (f, L)-almost $F$ contraction with applications," Journal of Nonlinear Science and Applications, vol. 9, pp. 1702-1715, 2016.

[13] M. Arshad, E. Ameer, and A. Hussain, "Hardy-Rogers-Type fixed point theorems for $\alpha$-GF-contractions," Archivum Mathematicum (BRNO) Tomus, vol. 51, pp. 129-141, 2015.

[14] M. Arshad, A. Shoaib, and P. Vetro, "Common fixed points of a pair of Hardy Rogers type mappings on a closed ball in ordered dislocated Metric Spaces," Journal of Function Spaces and Applications, vol. 2013, Article ID 638181, 9 pages, 2013.

[15] N. Boonsri, S. Chotchaisthit, and S. Saejung, "Common fixed point theorems for a Ciric-Reich-Rus pair of mappings in metric space with a directed graph," International Journal of Pure and Applied Mathematics, vol. 94, pp. 45-53, 2014.

[16] A. Shoaib, M. Arshad, E. Ameer, and A. Asif, "Generalized dynamic process for generalized multivalued $F$-contraction of Hardy Rogers type in b-metric spaces," Turkish Journal of Analysis and Number Theory, vol. 6, pp. 43-48, 2018.

[17] D. Wardowski, "Fixed points of a new type of contractive mappings in complete metric spaces," Fixed Point Theory and Applications, vol. 94, 2012.

[18] M. Cosentino and P. Vetro, "Fixed point results for F-contractive mappings of Hardy-Rogers type," Filomat, vol. 28, no. 4, pp. 715-722, 2014.

[19] M. Cosentino, M. Jleli, B. Samet, and C. Vetro, "Solvability of integro differential problems via fixed point theory in $b$ metric spaces," Fixed Point Theory and Applications, vol. 70, 2015.

[20] S. Kesornprom and P. Cholamjiak, "Proximal type algorithms involving linesearch and inertial technique for split variational inclusion problem in hilbert spaces with applications," Optimization, vol. 68, pp. 2365-2391, 2019.

[21] D. V. Thong and P. Cholamjiak, "Strong convergence of a forward-backward splitting method with a new step size for solving monotone inclusions," Computational and Applied Mathematics, vol. 38, p. 94, 2019.
[22] H. R. Marasi and H. Aydi, "Existence and uniqueness results for two-term nonlinear fractional differential equations via a fixed point technique," Journal of Mathematics, vol. 2021, Article ID 6670176, 7 pages, 2021.

[23] M. U. Ali, H. Aydi, and M. Alansari, "New generalizations of set valued interpolative Hardy-Rogers type contractions in $b$ metric spaces," Journal of Function Spaces, vol. 2021, Article ID 6641342, 8 pages, 2021.

[24] A. Asif, S. U. Khan, T. Abdeljawad, M. Arshad, and A. Ali, "3D dynamic programming approach to functional equations with applications," Journal of Function Spaces, vol. 2020, Article ID 9485620, 9 pages, 2020.

[25] A. Asif, S. U. Khan, T. Abdeljawad, M. Arshad, and E. Savas, "3D analysis of modified F-contractions in convex b-metric spaces with application to Fredholm integral equations," AIMS Mathematics, vol. 5, no. 6, pp. 6929-6948, 2020. 